\title{
An easy method for calculating kinetic parameters of electrochemical mechanisms: Temkin's formalism
}

\author{
F.J. Vidal-Iglesias*, J. Solla-Gullón, V. Montiel and A. Aldaz \\ Institute of Electrochemistry and Department of Physical Chemistry. University \\ of Alicante. \\ Email: fj.vidal@ua.es
}

\begin{abstract}
In this educational article the formalism developed by Temkin for a consecutive chemical mechanism is applied to work out the kinetic laws of consecutive electrochemical reaction mechanisms. The benefits of this approach will be highlighted in comparison with classical treatments such as the Quasi Equilibrium and the Steady State approximations. In particular, the use of this formalism becomes even more advantageous when the number of reaction steps increases.
\end{abstract}

\section{Introduction}

One of the typical problems addressed in electrochemical textbooks is how to define the theoretical kinetic law of an electrochemical reaction and how to propose a plausible mechanism for this reaction from its kinetic parameters, usually the Tafel slope and reaction orders.

Most electrochemical reactions consist of at least two electron transfers and it is usually accepted that their probability of being multiple simultaneous electron transfers is low, i.e. only single electron transfer reactions are possible (although it seems that simultaneous two electron transfer can occur in certain cases). Thus, for an n-electron transfer reaction such as

$$
R f O x+n e
$$

several mechanisms with different rate determining steps, rds, and different intermediates are possible.

The simplest two electron homogeneous redox process is composed of two single electron transfer steps and two diffusion steps, the diffusion of the reactant from the bulk solution to the electrode surface and the diffusion of the product from the electrode 
surface to the bulk solution (diffusion of the intermediate compounds is not taken into account).

After the reactant accepts (cathodic process) or loses (anodic process) one electron, the intermediate compound formed in this first electron transfer can be involved in different chemical reactions before the second electron transfer takes place. Thus, the number and complexity of the possible mechanisms rises with the increasing number of electrons transferred.

The typical approaches used for calculating the kinetic law of these mechanisms are steady-state approximation (SSA) and quasi-equilibrium approximation (QEA). More solid and precise physicochemical conclusions are obtained from the first approach; on the other hand it calls for more complicated algebraic manipulations, especially for mechanisms with a considerable number of steps and with kinetic orders higher than one.

\section{Steady-State approximation}

In this approximation it is assumed that after a certain time, induction time, the concentration of any intermediate, $\mathrm{B}$, will be constant which means that the rate of its appearance and disappearance will be equal, i.e $\left(\frac{d C_{B}}{d t}\right)=0$

\section{Quasi Equilibrium Approximation}

This approximation accepts that there is only one step which determines the reaction rate and that the other steps are very quick and at equilibrium. Logically, this step is called the rate determining step, rds. It should be never called the slowest step because at stationary state, the rate of all the steps is the same. This approximation is the most widely used in electrochemical textbooks due its simplicity and the use of very easy algebraic manipulations. In spite of the fact that this approximation seems very rough, one is likely to observe a rds, at least in a certain potential window, because electrochemical rate constants are functions of potential and do not vary in the same way. However, students should be made aware of the different approximations involved in the calculus: 
i) After an induction time, the reaction must reach a stationary state and there is only one step, that is taken as irreversible, that controls the velocity of the overall reaction.

ii) The surface concentration of the reactants is, at any time, equal to their bulk concentrations, i.e, the mass transfer is very quick.

iii) All the other steps are reversible and, potentially, very rapid, i.e, all of them have the same rate but their rate constants in both senses are at least 50-100 times bigger than that of the constant rate of the determining step.

iv) Besides, it should also be pointed out that because electrochemical rate constants depend on potential, and may not be in the same way for the different transfer steps, the rds could change with potential; that is to say, a step that is the rds at a given potential could not be the rds at another potential. This also means that the Tafel slope of the mechanism can change not only because different steps can have different symmetry coefficients, $\beta$, but also because the position of the rds can change.

v) It should be also pointed out to the students that there are limits to the overpotential window from which mechanistically significant Tafel slopes can be obtained. The diffusion processes do not have an infinite rate and when the current increases the surface concentration of the species that takes part in the reaction can be different to that of their bulk concentrations.

vi) The quasi-equilibrium approximation fails, for example, if there are two steps with similar low constant rate.

\section{Temkin's formalism}

The complexity of the calculus of the kinetic law for mechanisms that have a great number of steps has induced workers in chemical kinetics to try to shorten the algebraic procedure needed to work out the kinetic law of these mechanisms. Thus, in 1936 Christiensen [1] proposed a formula for a single stationary reaction with a linear mechanism that was later generalized by Temkin [2] to a non-linear situation. The method developed by this last author is now known as Temkin's formalism. This formalism allows the kinetic law of any mechanism to be defined very quickly and easily using elemental algebraic manipulations as has been shown by Boudart et al. [3] for consecutive mechanisms in stationary state. It is also possible to apply this 
formalism to reactions with steps of kinetic orders higher than 1 but, in this case, working out the kinetic parameters is a more complicated process.

In a consecutive mechanism, the overall reaction is the result of a linear combination of steps. The addition of these steps multiplied by an adequate factor must give the overall reaction without any intermediates. For example, let us take a reaction such as the electrochemical oxidation of hydrogen, the overall reaction of which is:

$$
H_{2} € 2 H^{+}+2 e^{-}
$$

and that on low overvoltage metals such as Pt, follows the so-called Tafel-Volmer mechanism:

$$
\begin{aligned}
& H_{2}+2 M f 2 M H \\
& M H f H^{+}+e^{-}
\end{aligned}
$$

where $\mathrm{M}$ is an adsorption site on the electrode surface and $\mathrm{MH}$ a hydrogen atom adsorbed on this site.

In order to work out the overall reaction from these two steps, the necessary factors to be used are 1 for the first step and 2 for the second one. In more kinetic terms, the number of times each step takes part in the overall process is different, being 2 for the second step and one for the first one. This number is called, according to Horiuti, the stoichiometric number of the step, $\sigma$.

For the redox couple $\mathrm{I}^{-} / \mathrm{I}_{2}$ with high iodine concentration, the mechanism proposed by Vetter [4], is

$$
\begin{aligned}
& I^{-} f \quad I+e \quad \sigma_{1}=2 \\
& \text { 2If } I_{2} \quad \sigma_{2}=1 \\
& I_{2}+I^{-} f \quad I_{3}^{-} \quad \sigma_{3}=1 \\
& 3 I^{-} f I_{3}^{-}+2 e \text { overall reaction }
\end{aligned}
$$

Thus, step 1 must be repeated twice in order to obtain the overall reaction and for this reason its stoichiometric number is two.

For a single route electrochemical mechanism with $\mathrm{i}$ steps and different stoichiometric numbers, such as:

$$
\begin{array}{lll}
\text { Af } & B+e & \sigma_{1} v=\left(v_{1}-v_{-1}\right) \\
\text { Bf } & C+e & \sigma_{2} v=\left(v_{2}-v_{-2}\right) \\
\text { ……......... } & \\
\text { Nf } & O+e & \sigma_{i} v=\left(v_{i}-v_{-i}\right)
\end{array}
$$


where $\sigma_{i}$ is the stoichiometric number of step i, $v_{i}$ and $v_{-i}$ the rates of the forward and backward reactions of step i and $v$ the overall reaction rate, the rate constants of step i, $k_{i}$ and $k_{-i}$, are related to potential by:

$$
k_{i}=k_{i}^{o} \exp \left(\left(1-\beta_{i}\right) f\left(E-E_{i}^{o}\right)\right)
$$

for the anodic process and

$$
k_{-i}=k_{i}^{o} \exp \left(-\beta_{i} f\left(E-E_{i}^{o}\right)\right)
$$

for the cathodic one; $k_{i}^{o}, E_{i}^{o}$ and $\beta_{i}$ are, respectively, the standard rate constant, the standard electrode potential and the symmetry coefficient of step i; $f$ stands for $f=\frac{F}{R T}$.

As indicated by Boudart et al. [3] if the reaction is at stationary state, all steps must proceed at the same rate and the global rate will be

$$
v=\frac{v_{1}-v_{-1}}{\sigma_{1}}=\frac{v_{2}-v_{-2}}{\sigma_{2}}=\ldots \frac{v_{i}-v_{-i}}{\sigma_{i}}
$$

and the following expression is worked out [3]:

$$
\begin{aligned}
& \prod_{i=1}^{i=n} v_{i}-\prod_{i=1}^{i=n} v_{-i}=\left(v_{1}-v_{-1}\right) v_{2} \cdot v_{3} \ldots v_{n}+v_{-1}\left(v_{2}-v_{-2}\right) v_{3} \ldots v_{n}+v_{-1} v_{-2}\left(v_{3}-v_{-3}\right) \ldots v_{n}+ \\
& +v_{-1} v_{-2} v_{-3} \ldots v_{-(n-1)}\left(v_{n}-v_{-n}\right)
\end{aligned}
$$

By opening the parentheses it is easy to check that because $v \sigma_{i}=\left(v_{i}-v_{-i}\right)$, can be expressed as:

$$
\prod_{i=1}^{i=n} v_{i}-\prod_{i=1}^{i=n} v_{-i}=v\left(\sigma_{1} v_{2} . v_{3} \ldots v_{n}+v_{-1} \sigma_{2} v_{3} \ldots v_{n}+v_{-1} v_{-2} \sigma_{3} \ldots v_{n}+v_{-1} v_{-2} v_{-3} \ldots v_{-(n-1)} \sigma_{n}\right)
$$

and

$$
v=\frac{\prod_{i=1}^{i=n} v_{i}-\prod_{i=1}^{i=n} v_{-i}}{\left(\sigma_{1} v_{2} . v_{3} \ldots v_{n}+v_{-1} \sigma_{2} v_{3} \ldots v_{n}+v_{-1} v_{-2} \sigma_{3} \ldots v_{n}+v_{-1} v_{-2} v_{-3} \ldots v_{-(\mathrm{n}-1)} \sigma_{n}\right)}
$$


As far as we are aware, and in spite of its simplicity, this formalism, has never been used in electrochemical textbooks. Only in one research article by Bockris [5] and in the books Theory and principles of Electrode processes [6] and Surface Electrochemistry [7], Christiensen's approach, which is restricted to linear steps with stoichiometric number equal to 1 , has been employed.

\section{Application of Temkin's formalism to different mechanisms}

Let us take a two step mechanism such as

$$
\begin{array}{lll}
\text { Af } & B+e & \sigma_{1}=1 \\
\text { Bf } & C+e & \sigma_{2}=1
\end{array}
$$

The use of the different approximations for working out the kinetic law, gives:

\section{1) Quasi equilibrium state approximation}

If step 2 is the rds, and step 1 is at equilibrium and accepting that mass transport is very rapid, i.e. the concentrations in the bulk solution and on the electrode surface are the same, $C^{\text {surf }}=C^{\text {bulk }}$, the rate of the reaction will be:

$$
v=k_{2} C_{B}-k_{-2} C_{C}
$$

If $C_{C}^{\text {bulk }}=0$, the anodic rate will be:

$$
v=k_{2} C_{B}
$$

Because step 1 is at equilibrium,

$$
\begin{aligned}
& k_{1} C_{A}=k_{-1} C_{B} \quad C_{B}=\frac{k_{1}}{k_{-1}} C_{A} \\
& k_{1}=k_{1}^{o} \exp \left(\left(1-\beta_{1}\right) f\left(E-E_{1}^{o}\right)\right) \\
& k_{-1}=k_{1}^{o} \exp \left(\left(-\beta_{1}\right) f\left(E-E_{1}^{o}\right)\right) \\
& C_{B}=C_{A} \exp \left(f\left(E-E_{1}^{o}\right)\right) \\
& v=k_{2} C_{B}=k_{2} C_{A} \exp \left(f\left(E-E_{1}^{o}\right)\right)= \\
& =C_{A} \exp \left(f\left(E-E_{1}^{o}\right)\right) \cdot k_{2}^{o} \exp \left(\left(1-\beta_{2}\right) f\left(E-E_{2}^{o}\right)\right)= \\
& =k_{2}^{o} C_{A} \exp \left(\left(2-\beta_{2}\right) f E-\left(\left(1-\beta_{2}\right) f E_{2}^{o}\right)-f E_{1}^{o}\right) \\
\mathrm{j}=2 \mathrm{Fv} v= & 2 F k_{2}^{o} C_{A} \exp \left(\left(2-\beta_{2}\right) f E-\left(\left(1-\beta_{2}\right) f E_{2}^{o}\right)-f E_{1}^{o}\right)
\end{aligned}
$$

Since $E_{2}^{o}$ and $E_{1}^{o}$ are constants, the Tafel slope at $25^{\circ} \mathrm{C}$ will be: 


$$
\left(\frac{\partial E}{\partial \log j}\right)_{C_{A}}=\frac{59}{\left(2-\beta_{2}\right)}=39 \mathrm{mV} \text { at } 25^{\circ} \mathrm{C} \text { for } \beta_{2}=0.5
$$

and the electrochemical reaction order with respect to reactant $A$, will be

$$
\left(\frac{\partial \log j}{\partial \log C_{A}}\right)_{E}=1
$$

\section{2) Steady state approximation.}

The reaction rate will be as before:

$$
v=k_{2} C_{B}-k_{-2} C_{C}
$$

and the value of $C_{B}$ can be deduced accepting that, at steady state, $\left(\frac{d C_{B}}{d t}\right)=0$

$$
\begin{aligned}
& \left(\frac{d C_{B}}{d t}\right)=k_{1} C_{A}-k_{-1} C_{B}-k_{2} C_{B}+k_{-2} C_{C}=0 \\
& C_{B}=\frac{k_{1} C_{A}+k_{-2} C_{C}}{k_{-1}+k_{2}}
\end{aligned}
$$

So that,

$$
v=k_{2} C_{B}-k_{-2} C_{C}=k_{2} \frac{k_{1} C_{A}+k_{-2} C_{C}}{k_{-1}+k_{2}}-k_{-2} C_{C}
$$

For $C_{C}=0$ :

$$
v=k_{2} \frac{k_{1} C_{A}}{k_{2}+k_{-1}}
$$

The rate constants $k_{i}$ and $k_{-i}$ depend on potential:

$$
\begin{aligned}
& k_{i}=k_{i}^{o} \exp \left((1-\beta) f\left(E-E^{o}\right)\right) \\
& k_{-i}=k_{i}^{o} \exp \left(-\beta f\left(E-E^{o}\right)\right)
\end{aligned}
$$


and

$$
v=k_{2}^{o} \exp \left(\left(1-\beta_{2}\right) f\left(E-E_{2}^{o}\right)\right) \frac{k_{1}^{o} \exp \left(\left(1-\beta_{1}\right) f\left(E-E_{1}^{o}\right)\right) \cdot C_{A}}{k_{2}^{o} \exp \left(\left(1-\beta_{2}\right) f\left(E-E_{2}^{o}\right)\right)+k_{1}^{o} \exp \left(-\beta_{1} f\left(E-E_{1}^{o}\right)\right)}
$$

From this equation, only the reaction order with respect to $C_{A}$ can easily be obtained but not the Tafel slope which is a complicated function of E. Only, if we accept that either the constant of the forward rate of step 2 is very small, $k_{2}<<k_{-1}$ i.e the rds is step 2, or the backward constant of step 1 is very small, $k_{2}>>k_{-1}$ i.e., step 1 is the rds, can we obtain equations that are identical to those obtained using the QEA for these two cases.

However, it might be pointed out to students that for the QEA, more restrictive conditions are employed. For example if step 2 is the rds, step 1 must be in equilibrium with very high rate constants $k_{i}$ and $k_{-i}$. This is not a restrictive condition for the SSA because $k_{i}$ does not need to be very high.

\section{Temkin's formalism.}

Applying (5) to the mechanism (6):

$$
v=\frac{v_{1} v_{2}-v_{-1} v_{-2}}{\sigma_{1} v_{2} \cdot+\sigma_{2} v_{-1}}=\frac{k_{1} k_{2} C_{A} C_{B}-k_{-1} k_{-2} C_{B} C_{C}}{k_{2} C_{B}+k_{-1} C_{B}}=\frac{k_{1} k_{2} C_{A}-k_{-1} k_{-2} C_{C}}{k_{2}+k_{-1}}
$$

If the bulk concentration of $\mathrm{C}$ is zero:

$$
v=\frac{k_{1} k_{2} C_{A}}{k_{2}+k_{-1}}
$$

This is the same equation as was obtained from the SSA but with the advantage that to achieve the result it was not necessary to resolve any differential equation which in this case is not cumbersome, but it can be if the number of steps increases.

Obviously for either $k_{2}>>k_{-1}$ or for $k_{2}<<k_{-1}$ the same equations as those that obtained for the SSA are achieved. Thus, for $k_{2}<<k_{-1}$, the expression for the current density will be:

$$
\begin{aligned}
& j=2 F v=2 F \frac{k_{1} k_{2} C_{A}}{k_{-1}}=2 F \exp \left(f\left(E-E_{1}^{o}\right)\right) \cdot k_{2}^{o} \exp \left(\left(1-\beta_{2}\right) f\left(E-E_{2}^{o}\right)\right) C_{A} \\
& \mathrm{j}=2 \mathrm{~F} v=2 F k_{2}^{o} C_{A} \exp \left(\left(2-\beta_{2}\right) f E-\left(\left(1-\beta_{2}\right) f E_{2}^{o}\right)-f E_{1}^{o}\right)
\end{aligned}
$$


From this expression, the calculated Tafel slope and reaction order respect $\mathrm{C}_{\mathrm{A}}$ are the same than those obtained for the QEA:

The only apparent difference between the SSA and Temkin's formalism, is that one might think that using the formalism, the concentration of the intermediate B can not be obtained, but this is not true. Following Temkin's reasoning we can always write an identity for the reaction rates that remains valid whatever the ordering of the steps. Thus, if instead of the mechanism:

$$
\begin{array}{llll}
\text { Af } & B+e & \sigma_{1}=1 & \text { step } 1 \\
\text { Bf } & C+e & \sigma_{2}=1 & \text { step } 2
\end{array}
$$

we use:

$$
\begin{array}{llll}
\text { Bf } & C+e & \sigma_{2}=1 & \text { step } 2 \\
\text { Af } & \text { B }+e & \sigma_{1}=1 & \text { step } 1
\end{array}
$$

and we apply the formalism to this last sequence of steps, then:

$$
v=\frac{v_{1} v_{2}-v_{-1} v_{-2}}{\sigma_{1} v_{2}+\sigma_{2} v_{-1}}
$$

Since the overall rate must be the same:

$$
\begin{aligned}
& v=\frac{v_{1} v_{2}-v_{-1} v_{-2}}{\sigma_{2} v_{1}+\sigma_{1} v_{-2}}=\frac{v_{1} v_{2}-v_{-1} v_{-2}}{\sigma_{1} v_{2}+\sigma_{2} v_{-1}} \\
& v_{1}+v_{-2}=v_{2}+v_{-1} \\
& k_{1} C_{A}+k_{-2} C_{c}=k_{2} C_{B}+k_{-1} C_{B} \\
& C_{B}=\frac{k_{1} C_{A}+k_{-2} C_{c}}{k_{2}+k_{-1}}
\end{aligned}
$$

that is the same expression as that obtained using the SSA.

Temkin's formalism can also be used to show students how a general expression for the relation of current density to potential, $j=f(E)$, can be obtained without any initial hypothesis, as for example, that the reaction is only controlled either by diffusion or by a charge transfer.

Let us take a mechanism such as:

$$
\begin{array}{lll}
\text { 1step } & R^{\text {bulk }} f \quad R^{\text {surf }} \\
\text { 2step } & R^{\text {surf }} f \quad O x^{\text {surf }}+e \\
\text { 3step } & O x^{\text {surf }} f \quad O x^{\text {bulk }}
\end{array}
$$


with the same rate constants $k_{D}$, for both diffusion processes, i.e. both species have the same diffusion coefficient, and rate constants $k_{i}$ and $k_{-i}$ for the charge transfer process. Because the reaction can go in both directions, anodic and cathodic, we can accept that the diffusion steps are reversible in the sense that the mass flux of $\mathrm{R}$ and $\mathrm{O}$ has opposite signs for the anodic and cathodic processes:

For the anodic direction,

$$
\begin{aligned}
& R^{\text {bulk }} \rightarrow R^{\text {surf }} \\
& O x^{\text {surf }} \rightarrow O x^{\text {bulk }}
\end{aligned}
$$

and for the cathodic one

$$
\begin{aligned}
& O x^{\text {bulk }} \rightarrow O x^{\text {surf }} \\
& R^{\text {surf }} \rightarrow R^{\text {bulk }}
\end{aligned}
$$

Thus, applying the formalism to the mechanism:

$$
v=\frac{v_{1} v_{2} v_{3}-v_{-1} v_{-2} v_{-3}}{\sigma_{1} v_{2} \cdot v_{3}+\sigma_{2} v_{-1} v_{3}+\sigma_{3} v_{-1} v_{-2}}
$$

If for the anodic process we accept that $C_{O x}^{b u l k}=0, v_{-3}$ will be 0 and:

$$
v=\frac{v_{1} v_{2} v_{3}}{v_{2} \cdot v_{3}+v_{-1} v_{3}+v_{-1} v_{-2}}=\frac{k_{1} k_{2} k_{3} C_{R}^{\text {bulk }} C_{R}^{\text {surf }} C_{O x}^{\text {surf }}}{k_{2} k_{3} C_{R}^{\text {surf }} C_{O x}^{\text {surf }}+k_{-1} k_{3} C_{R}^{\text {surf }} C_{O x}^{\text {surf }}+k_{-1} k_{-2} C_{R}^{\text {surf }} C_{O x}^{\text {surf }}}
$$

Because the two diffusion rate constants are the same $k_{1}=k_{-1}=k_{3}=k_{-3}=k_{D}$

$$
j=F V=F \frac{k_{D}^{2} k_{2} C_{R}^{\text {bulk }}}{k_{2} k_{D}+k_{D}^{2}+k_{D} k_{-2}}=F \frac{k_{D}}{\left(1+\frac{k_{D}}{k_{2}}+\frac{k_{-2}}{k_{2}}\right)} C_{R}^{\text {bulk }}
$$

The rate constants $k_{2}$ and $k_{-2}$ depend on the electrode potential according to

$$
\begin{aligned}
& k_{2}=k^{o} \exp \left((1-\beta) f\left(E-E^{o}\right)\right) \\
& k_{-2}=k^{o} \exp \left(-\beta f\left(E-E^{o}\right)\right)
\end{aligned}
$$

For a very positive value of $E$ (large anodic overvoltage) $k_{2}>k_{-2}$ and $k_{2}>>k_{D}$ and (14) becomes:

$$
j_{l}=F k_{D} C_{R}^{b u l k}
$$

and the current density reaches its maximum value, known as limiting diffusion current $j_{l}$, which does not depend on electrode potential. Using this limiting diffusion current in expression (14) and rearranging: 


$$
\frac{j_{l}}{j}-1=\frac{k_{D}}{k_{2}}+\frac{k_{-2}}{k_{2}}=\frac{k_{D}}{k^{o} \exp \left((1-\beta) f\left(E-E^{o}\right)\right)}+\exp \left(-f\left(E-E^{o}\right)\right)
$$

If $k^{o}$ is very high, reversible process, the first term on the right-hand equation can be neglected and equation (16) is transformed into

$$
E=E^{o}-\frac{1}{f} \ln \left(\frac{j_{l}-j}{j}\right)=E^{o}-\frac{R T}{F} \ln \left(\frac{j_{l}-j}{j}\right)
$$

which is the classical expression for a reversible mono-electron oxidation process, $f=\frac{F}{R T}$.

If the ratio $k_{D} / k^{o}$ is very high, i.e. $k^{o}$ small, the term $\exp \left(-f\left(E-E^{o}\right)\right)$ can be neglected, and equation [16] is transformed into

$$
E=E^{o}+\frac{R T}{F(1-\beta)} \ln \left(\frac{k_{D}}{k^{o}}\right)-\frac{R T}{F(1-\beta)} \ln \left(\frac{j_{l}-j}{j}\right)
$$

which corresponds to a totally irreversible mono-electron oxidation process.

It should be pointed out that the algebraic process for working out the global expression (16) has been very easy and that the values of the intermediate concentrations $C_{R}^{\text {surf }}$ and $C_{O x}^{\text {surf }}$ were not used.

To work out the same equations using the SSA, the procedure should be:

Working out the differential equations for the steady state:

$$
\begin{aligned}
& \left(\frac{d C_{R}^{\text {surf }}}{d t}\right)=k_{1} C_{R}^{\text {bulk }}-k_{-1} C_{R}^{\text {surf }}-k_{2} C_{R}^{\text {surf }}+k_{-2} C_{O x}^{\text {surf }}=0 \\
& \left(\frac{d C_{O x}^{\text {surf }}}{d t}\right)=k_{2} C_{R}^{\text {surf }}-k_{-2} C_{O x}^{\text {surf }}-k_{3} C_{O x}^{\text {surf }}+k_{-3} C_{O x}^{\text {bulk }}=0
\end{aligned}
$$

Finding the concentrations of the intermediates from this equation system (their values must be known to get the final solution).

$$
C_{R}^{\text {surf }}=\frac{\left(1+\frac{k_{-2}}{k_{D}}\right)}{\left(1+\frac{k_{2}}{k_{D}}+\frac{k_{-2}}{k_{D}}\right)} C_{R}^{\text {bulk }}
$$

and 


$$
C_{O x}^{\text {surf }}=\frac{\left(\frac{k_{2}}{k_{D}}\right)}{\left(1+\frac{k_{2}}{k_{D}}+\frac{k_{-2}}{k_{D}}\right)} C_{R}^{\text {bulk }}
$$

Defining the current density of the process by

$$
j=F\left(k_{2} C_{R}^{\text {surf }}-k_{-2} C_{O x}^{\text {surf }}\right)
$$

and after some algebraic manipulations, the expression

$$
j=F \frac{k_{D}}{\left(1+\frac{k_{D}}{k_{2}}+\frac{k_{-2}}{k_{2}}\right)} C_{R}^{\text {bulk }}
$$

is obtained. Obviously, this procedure is more complicated than that of Temkin's formalism.

If for any reason, the concentrations of the intermediates are needed, they can be obtained using the same reasoning as before: we can always write an identity for the reaction rates that is valid whatever the ordering of the steps.

Thus, for sequence 1-3-2

$$
v=\frac{v_{1} v_{2} v_{3}}{v_{3} \cdot v_{2}+v_{-1} v_{2}+v_{-1} v_{-3}}=\frac{v_{1} v_{2} v_{3}}{v_{2} \cdot v_{3}+v_{-1} v_{2}}
$$

For sequence 2-1-3

$$
v=\frac{v_{1} v_{2} v_{3}}{v_{1} v_{3}+v_{-2} v_{3}+v_{-1} v_{-2}}
$$

For sequence 2-3-1

$$
v=\frac{v_{1} v_{2} v_{3}}{v_{3} v_{1}+v_{-2} v_{1}+v_{-2} v_{-3}}=\frac{v_{1} v_{2} v_{3}}{v_{3} v_{1}+v_{-2} v_{1}}
$$

For sequence 3-2-1

$$
v=\frac{v_{1} v_{2} v_{3}}{v_{2} v_{1}}
$$

For sequence 3-1-2

$$
v=\frac{v_{1} v_{2} v_{3}}{v_{2} v_{1}}
$$


Because the reaction rate $v$ is the same for all sequences:

For 1-2-3 and 1-3-2

$$
\begin{aligned}
& v_{-1} v_{3}+v_{-1} v_{-2}=v_{-1} v_{2} \\
& v_{3}+v_{-2}=v_{2}
\end{aligned}
$$

For 1-2-3 and 2-1-3

$$
\begin{aligned}
& v_{2} v_{3}+v_{-1} v_{3}=v_{1} v_{3}+v_{-2} v_{3} \\
& v_{2}+v_{-1}=v_{1}+v_{-2}
\end{aligned}
$$

For 1-3-2 and 3-1-2

$$
\begin{aligned}
& v_{3} v_{2}+v_{-1} v_{2}=v_{1} v_{2} \\
& v_{3}+v_{-1}=v_{1}
\end{aligned}
$$

Sequences 2-1-3 and 2-3-1 give the same result as that of sequences 1-3-2 and 31-2, and the same happens for sequences 2-3-1 and 3-2-1 compared to 1-2-3 and 1-3-2.

Taking into account that

$$
\begin{gathered}
v_{1}=k_{D} C_{R}^{\text {bulk }} ; v_{-1}=k_{D} C_{R}^{\text {surf }} ; v_{2}=k_{2} C_{R}^{\text {surf }} ; v_{-2}=k_{-2} C_{O x}^{\text {surf }} ; v_{3}=k_{D} C_{O x}^{\text {surf }} ; v_{-3}=k_{D} C_{O x}^{\text {bulk }} \\
k_{2} C_{R}^{\text {surf }}=k_{D} C_{O x}^{\text {surf }}+k_{-2} C_{O x}^{\text {surf }} \\
k_{2} C_{R}^{\text {surf }}+k_{D} C_{R}^{\text {surf }}=k_{D} C_{R}^{\text {bulk }}+k_{-2} C_{O x}^{\text {surf }} \\
k_{D} C_{O x}^{\text {surf }}+k_{D} C_{R}^{\text {surf }}=k_{D} C_{R}^{\text {bulk }}
\end{gathered}
$$

Substituting $C_{O x}^{\text {surf }}$ in (22)

$$
\begin{aligned}
& \left(\frac{k_{D} k_{2}}{k_{D}+k_{-2}}\right) C_{R}^{\text {surf }}+k_{D} C_{R}^{\text {surf }}=k_{D} C_{R}^{\text {bulk }} \\
& C_{R}^{\text {surf }}=\frac{\left(1+\frac{k_{-2}}{k_{D}}\right)}{\left(1+\frac{k_{2}}{k_{D}}+\frac{k_{-2}}{k_{D}}\right)} C_{R}^{\text {bulk }}
\end{aligned}
$$

In the same way: 


$$
C_{O x}^{\text {surf }}=\frac{\left(\frac{k_{2}}{k_{D}}\right)}{\left(1+\frac{k_{2}}{k_{D}}+\frac{k_{-2}}{k_{D}}\right)} C_{R}^{\text {bulk }}
$$

\section{Conclusion}

The formalism developed by Temkin can be used quite easily to work out the kinetic law of a consecutive mechanism of electrochemical reactions and also to work out general electrochemical equations. We believe that it can also help students to understand that when they use the QEA there are a lot of hidden assumptions that should be taken into account when plausible mechanism must be obtained from experimental data.

\section{Acknowledgements}

This work has been financially supported by the MEC (Spain) through CTQ201348280-C3-3-R project. We thank Prof. Ángela Molina for her helpful comments.

\section{Bibliography}

[1] J.A. Christiansen, Z Physik. Chem. B33, 145 (1936)

[2] M.I. Temkin Int. Chem. Eng. 11 (4), 709 (1971)

[3] M. Boudart, G. Djéga-Mariadassou, Kinetics of Heterogeneous Catalytic

Reactions, Princeton University Press (1984)

[4] K.J. Vetter Electrochemical kinetics, Theoretical and experimental aspects, Academic Press (1967)

[5] J.O’M Bockris J. Phys. Chem. 24, 817 (1956)

[6] B.E. Conway, Theory and principles of Electrode processes, Ronald Press (1965)

[7] J.O’M Bockris and S.U.M.Khan, Surface Electrochemistry: a molecular level approach, Plenum Press (1993) 